\title{
Max-Min Lyapunov Functions for Switching Differential Inclusions
}

Matteo Della Rossa

\author{
Aneel Tanwani
}

\author{
Luca Zaccarian
}

\begin{abstract}
We use a class of locally Lipschitz continuous Lyapunov functions to establish stability for a class of differential inclusions where the set-valued map on the right-handside comprises the convex hull of a finite number of vector fields. Starting with a finite family of continuously differentiable positive definite functions, we study conditions under which a function obtained by max-min combinations over this family of functions is a Lyapunov function for the system under consideration. For the case of linear systems, using the $S$ Procedure, our conditions result in bilinear matrix inequalities. The proposed construction also provides nonconvex Lyapunov functions, which are shown to be useful for systems with statedependent switching that do not admit a convex Lyapunov function.
\end{abstract}

\section{INTRODUCTION}

The construction of Lyapunov functions is one of the central ingredients in the stability analysis of switching dynamical systems, or hybrid systems, and several approaches exist in the literature to address this problem. In this paper, we are interested in providing a procedure for the construction of common Lyapunov functions for systems which involve switching among several vector fields.

For the system class we are interested in, let us consider a finite number of dynamical subsystems described by ordinary differential equations (ODEs) of the form $\dot{x}=f_{i}(x)$, where $i \in\{1,2, \ldots, m\}$, and each $f_{i}: \mathbb{R}^{n} \rightarrow \mathbb{R}^{n}$ is locally Lipschitz continuous. To model the evolution of state-trajectories resulting from switching arbitrarily among these dynamical subsystems, we consider the differential inclusion (DI)

$$
\dot{x} \in \overline{\mathrm{co}}\left\{f_{i}(x) \mid i \in\{1, \ldots, m\}\right\}
$$

where $\overline{c 0}\{S\}$ denotes the closed convex-hull of the set $S$. The DI in (1) indeed results from an appropriate regularization of the switching dynamics (see Section V for some details). The problem of interest is to construct a Lyapunov functions for system (1) which guarantees stability of the origin $\{0\} \subset \mathbb{R}^{n}$.

For the linear differential inclusion (LDI) case (that is $f_{i}(x)=A_{i} x$ for some $\left.A_{i} \in \mathbb{R}^{n \times n}\right)$ it is shown in [1], [2] that asymptotic stability is equivalent to the existence of a common Lyapunov function that is convex, homogeneous of degree 2 , and $\mathcal{C}^{1}\left(\mathbb{R}^{n}, \mathbb{R}\right)$. Many ways to approximate this kind of functions have been studied, for example the maximum of quadratic functions and its convex conjugates [3], [4], and polyhedral functions [2], [5].

In this article, we propose another class of Lyapunov functions for system (1). We consider a finite family of continuously differentiable positive definite functions, and

The authors are with LAAS-CNRS, University of Toulouse, 7 Avenue du Colonel Roche, F-31400 Toulouse, France. Luca Zaccarian is also with Dipartimento di Ingegneria Industriale, University of Trento, Italy obtain a candidate Lyapunov function by taking the maximum, minimum, or the combination of both; see Definition 3 for details. Such max-min type of Lyapunov functions were recently proposed in the context of discrete-time switching systems [6], [7]. In this article, we investigate the feasibility and utility of max-min Lyapunov functions, for differential inclusion and switching systems in continuous-time, which naturally require certain additional tools from nonsmooth and set-valued analysis. Our main results provide a set of inequalities whose feasibility guarantees the existence of a max-min Lyapunov function for system (1). When restricting ourselves to the linear case with $f_{i}(x)=A_{i} x$, the proposed conditions require solving bilinear matrix inequalities (BMIs). It should be noted that, since we allow for the minimum operation in the construction, certain elements in our proposed class of Lyapunov functions are nonconvex. For the linear DI problem, it has been observed in [3, Proposition 2.2] that the convexification of any non-convex Lyapunov function is still a Lyapunov function. In our approach, when we construct a homogeneous of degree 2 nonconvex Lyapunov function for the LDI problem, a convexification of such functions also provides a Lyapunov function.

The situation is different when the system is embedded with a given switching function $\sigma: \mathbb{R}^{n} \rightarrow\{1, \ldots, m\}$, resulting in

$$
\dot{x}=f_{\sigma(x)}(x) .
$$

Indeed, it is possible that the switched system (2) is asymptotically stable but there does not exist a convex Lyapunov function, see [8]. It is possible to provide sufficient conditions for a minimum of quadratics (clearly non-convex) to be a Lyapunov function in this context [9], [10]. When addressing this system class, our approach provides a more general class of nonconvex Lyapunov functions.

\section{A Motivating Example}

To provide a motivation for the class of Lyapunov functions constructed in this paper, we consider a system for which we will construct a max-min Lyapunov function.

Example 1. Consider a linear switching system with three subsystems and a state-dependent switching rule $x \mapsto$ $\sigma(x) \in\{1,2,3\}$. We consider matrices

$$
A_{1}=\left[\begin{array}{cc}
-0.1 & 1 \\
-5 & -0.1
\end{array}\right], \quad A_{2}=\left[\begin{array}{cc}
-0.1 & 5 \\
-1 & -0.1
\end{array}\right], \quad A_{3}=\left[\begin{array}{cc}
1.9 & 3 \\
-3 & -2.1
\end{array}\right]
$$

and the system

$$
\dot{x}=A_{\sigma(x)} x .
$$




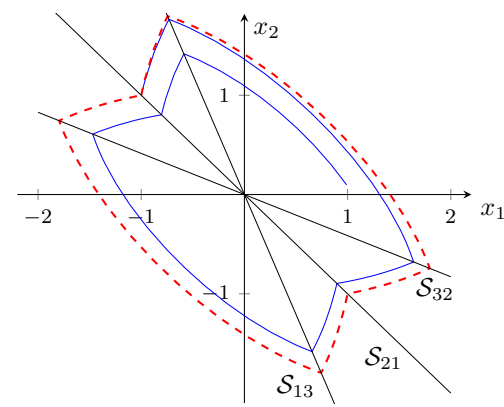

Fig. 1. The blue line shows a trajectory of system (3) starting from $z_{0}$. The dashed line indicates a level set of max-min Lyapunov function (4).

To suitably define switching signal $\sigma$, we introduce

$$
\begin{aligned}
Q_{1} & :=\left[\begin{array}{cc}
-\frac{1}{(1+\sqrt{2})} & -\frac{\sqrt{2}}{2} \\
-\frac{\sqrt{2}}{2} & -1
\end{array}\right], Q_{2}:=\left[\begin{array}{cc}
-(1+\sqrt{2}) & -\frac{2+\sqrt{2}}{2} \\
-\frac{2+\sqrt{2}}{2} & -1
\end{array}\right], \\
Q_{3} & :=\left[\begin{array}{cc}
1 & \sqrt{2} \\
\sqrt{2} & 1
\end{array}\right]
\end{aligned}
$$

and the switching signal

$$
\sigma(x)= \begin{cases}1 & \text { if } x \in \mathcal{S}_{1}:=\left\{x^{\top} Q_{1} x>0\right\} \cup \mathcal{S}_{13}, \\ 2 & \text { if } x \in \mathcal{S}_{2}:=\left\{x^{\top} Q_{2} x>0\right\} \cup \mathcal{S}_{21}, \\ 3 & \text { if } x \in \mathcal{S}_{3}:=\left\{x^{\top} Q_{3} x>0\right\} \cup \mathcal{S}_{32},\end{cases}
$$

where the switching surfaces $\mathcal{S}_{i j}, 1 \leq i, j \leq 3, i \neq j$ are

$$
\begin{aligned}
& \mathcal{S}_{13}:=\left\{x \in \mathbb{R}^{2} \mid x_{2}=-(1+\sqrt{2}) x_{1}\right\}, \\
& \mathcal{S}_{21}:=\left\{x \in \mathbb{R}^{2} \mid x_{2}=-x_{1}\right\}, \\
& \mathcal{S}_{32}:=\left\{x \in \mathbb{R}^{2} \mid x_{2}=-1 /(1+\sqrt{2}) x_{1}\right\} .
\end{aligned}
$$

We note that $\mathcal{S}_{1} \cup \mathcal{S}_{2} \cup \mathcal{S}_{3}=\mathbb{R}^{2}$ and $\mathcal{S}_{1} \cap \mathcal{S}_{2} \cap \mathcal{S}_{3}=\{0\}$.

A Poincaré type of reasoning suggests that the origin is asymptotically stable: the trajectories showed in Figure 1 are shield-shaped inward spirals. Moreover one can formally prove that there does not exist a convex Lyapunov function for system (3), see [11]. In Section V, we will provide a Lyapunov function for this system of the form

$$
V(x)=\max \left\{\min \left\{x^{\top} P_{1} x, x^{\top} P_{2} x\right\}, x^{\top} P_{3} x\right\} .
$$

\section{MAX-MIN LYAPUNOV FUNCTION}

We now address the problem of stability analysis for system (1). We first state some definitions and a known result, which are then used to state our first main result.

\section{A. Background and notation}

Definition 1. For system (1), with $f_{i}(0)=0$ for all $i \in$ $\{1, \ldots, m\}$, the origin $\{0\}$ is asymptotically stable (AS) if:

1) (Stability) For each $\varepsilon>0$ there exists $\delta(\varepsilon)>0$ such that for every solution $x(t)$ of (1) that satisfies $|x(0)|<\delta(\varepsilon)$, it holds that $|x(t)|<\varepsilon$ for all $t>0$;

2) (Attractivity) There exists $M>0$ such that for every solution $x(t)$ that satisfies $|x(0)|<M$, it holds that $\lim _{t \rightarrow \infty}|x(t)|=0$.

If property 2) is true for every $M>0$, then we say that $\{0\}$ is globally asymptotically stable (GAS).
It is well known that the asymptotic stability can be proved via Lyapunov-based techniques. Our proposed construction is based on functions that are not everywhere differentiable, so we need the following notion of generalized gradients.

Definition 2. Let $U: \mathbb{R}^{n} \rightarrow \mathbb{R}$ be a locally Lipschitz continuous function. The generalized directional derivative of $U$ at $x$ along $v \in \mathbb{R}^{n}$, denoted $U^{0}(x ; v)$, is defined as

$$
U^{0}(x ; v):=\limsup _{y \rightarrow x ; h \rightarrow 0^{+}} \frac{U(y+h v)-U(y)}{h} .
$$

We say that $\zeta \in \mathbb{R}^{n}$ belongs to the generalized gradient of $U$ at $x$, denoted $\zeta \in \partial U(x)$, if

$$
U^{0}(x ; v) \geq \zeta^{\top} v, \quad \forall v \in \mathbb{R}^{n} .
$$

It is obvious that if $U$ is continuously differentiable then $U^{0}(x ; v)=\nabla U(x)^{\top} v$ and $\partial U(x)=\nabla U(x)$.

Lemma 1. Suppose that there exist a locally Lipschitz function $V: \mathbb{R}^{n} \rightarrow \mathbb{R}$, and a class $\mathcal{K}$ function $^{1} \gamma$ such that

1) $V(0)=0$,

2) $V(x)>0$, for all $x \neq 0$,

3) For each $x \in \mathbb{R}^{n}$, and for each $i \in\{1, \ldots, m\}$,

$$
\sup _{\zeta \in \partial V(x)} \zeta^{\top} f_{i}(x) \leq-\gamma(|x|)
$$

Then the origin of system (1) is AS, and $V$ is called a Lyapunov function for (1). If, in addition, $V$ is radially unbounded, that is, $V(x) \rightarrow \infty$ if $|x| \rightarrow \infty$, then system (1) is GAS.

The above result relates asymptotic stability with the existence of a nonsmooth Lyapunov function. For the technical details regarding the generalized gradient and the proof of Lemma 1, we suggest [12, Proposition 5.3], [13]. We next propose a class of functions which under certain conditions will be shown to satisfy the hypotheses of Lemma 1.

Definition 3. Given $K$ functions $V_{1}, \ldots V_{K} \in \mathcal{C}^{1}\left(\mathbb{R}^{n}, \mathbb{R}\right)$, we define a max-min function $V_{\mathrm{Mm}}: \mathbb{R}^{n} \rightarrow \mathbb{R}$ as

$$
V_{\mathbf{M m}}(x):=\max _{j \in\{1, \ldots, J\}}\left\{\min _{k \in S_{j}}\left\{V_{k}(x)\right\}\right\},
$$

where $S_{1}, \ldots, S_{J}$ are subsets of $\{1, \ldots, K\}$, i.e. $S_{j} \subset$ $\{1, \ldots, K\}, \forall j \in\{1, \ldots, J\}$.

We will denote by $\operatorname{Mm}\left(V_{1}, \ldots, V_{K}\right)$ the set of all the possible max-min functions obtained from functions $V_{1}, \ldots, V_{K}$

Definition 4. Given $V \in \operatorname{Mm}\left(V_{1}, \ldots, V_{K}\right)$ we can construct a map $\alpha_{V}: \mathbb{R}^{n} \rightrightarrows\{1, \ldots, K\}$ defined as follows:

$\alpha_{V}(x):=\left\{\begin{array}{c}\ell \mid \forall \text { neighborhood } \mathcal{U} \text { of } x, \exists \mathcal{V} \subset \mathcal{U} \text { open } \\ \text { s.t. } V(z)=V_{\ell}(z), \forall z \in \mathcal{V}\end{array}\right\}$.

Intuitively the set-valued map $\alpha_{V}$ captures the fact that every point $x \in \mathbb{R}^{n}$ is "surrounded" by regions where the

\footnotetext{
${ }^{1}$ A function $\gamma: \mathbb{R}_{\geq 0} \rightarrow \mathbb{R}_{\geq 0}$ is of class $\mathcal{K}$ if $\gamma(0)=0, \gamma$ is continuous and increasing.
} 
function $V$ is continuously differentiable and equal to $V_{\ell}$, for some $\ell$. As an example, consider $P_{1}, P_{2}>0, P_{1} \neq P_{2}$, and the function $V(x)=\max \left\{x^{\top} P_{1} x, x^{\top} P_{2} x\right\}$, which leads to $\alpha_{V}(x)=\{1,2\}$ when $x^{\top} P_{1} x=x^{\top} P_{2} x$.

\section{B. Stability result}

Our goal is to provide conditions under which a max-min function of type (6) is a Lyapunov function for system (1).

Theorem 1. Consider the DI (1), and given $K$ positivedefinite functions $V_{1}, \ldots, V_{K} \in \mathcal{C}^{1}\left(\mathbb{R}^{n}, \mathbb{R}\right)$, consider a maxmin function $V \in \mathbf{M m}\left\{V_{1}, \ldots, V_{K}\right\}$. If there exists a class $\mathcal{K}$ function $\gamma$ such that for all $x \in \mathbb{R}^{n}$, and for all $i \in$ $\{1, \ldots, m\}$

$$
\nabla V_{\ell}(x)^{\top} f_{i}(x) \leq-\gamma(|x|), \quad \forall \ell \in \alpha_{V}(x),
$$

then (1) is AS and $V$ is a Lyapunov function for system (1). Moreover, if each $V_{j}$ is radially unbounded, then (1) is GAS.

Proof. First of all, from definition (6), it is seen that $V$ is positive-definite and $V(0)=0$. Furthermore, $V$ is locally Lipschitz continuous by construction and for such functions

$$
\partial V(x)=\overline{\mathrm{co}}\left\{\lim _{k \rightarrow \infty} \nabla V\left(x_{k}\right) \mid x_{k} \rightarrow x, x_{k} \notin \mathcal{N}, x_{k} \notin \mathcal{S}\right\},
$$

where $\mathcal{N} \subset \mathbb{R}^{n}$ is the set of zero measure where $\nabla V$ is not defined, and $\mathcal{S} \subset \mathbb{R}^{n}$ is any other set of measure zero; see [14, Theorem 2.5.1, on page 63]. Using (9), we will show that

$$
\partial V(x)=\overline{\operatorname{co}}\left\{\nabla V_{\ell}(x) \mid \ell \in \alpha_{V}(x)\right\},
$$

whence (5) in Lemma 1 follows. Indeed, if (10) holds, then for a given $x \in \mathbb{R}^{n}$, let us suppose that $\alpha_{V}(x)=$ $\left\{\ell_{1}, \ldots, \ell_{p}\right\}$. For each $v \in \partial V(x)$ there exist $\lambda_{1}, \ldots, \lambda_{p} \geq 0$, $\sum_{j=1}^{p} \lambda_{j}=1$, such that $v=\sum_{j=1}^{p} \lambda_{j} \nabla V_{\ell_{j}}(x)$. Consequently, for each $i \in\{1, \ldots, m\}$, (8) yields

$$
v^{\top} f_{i}(x)=\sum_{j=1}^{p} \lambda_{j} \nabla V_{\ell_{j}}(x)^{\top} f_{i}(x) \leq-\gamma(|x|) .
$$

Thus, under condition (8), $V \in \operatorname{Mm}\left\{V_{1}, \ldots, V_{K}\right\}$ satisfies the first three conditions listed in Lemma 1, which shows that $V$ is a Lyapunov function for system (1). For GAS, if every $V_{j}, j \in\{1, \ldots, K\}$, is radially unbounded, then so is $V$. To complete the proof, it remains to show that (9) implies (10). We study two cases to show this implication.

Case 1: Consider $\bar{x} \in \mathbb{R}^{n}$ such that $\alpha_{V}$ is constant in an open neighborhood $\mathcal{U}_{\bar{x}}$ of $\bar{x}$. Then, for each $x \in \mathcal{U}_{\bar{x}}$, $V_{\ell_{i}}(x)=V_{\ell_{j}}(x)$, for each $\ell_{i}, \ell_{j} \in \alpha_{V}(x)$. Since each $V_{j}$, $j \in\{1, \ldots, K\}$ is differentiable, we have that $\nabla V(x)=$ $\nabla V_{\ell_{i}}(x)$, for each $\ell_{i} \in \alpha_{V}(x)$, and $x \in \mathcal{U}_{\bar{x}}$. Thus, for each $x \in \mathcal{U}_{\bar{x}}$, (9) yields

$$
\partial V(x)=\nabla V(x)=\nabla V_{\ell}(x)
$$

for some $\ell \in \alpha_{V}(x)$.

Case 2: Let $\mathcal{S}$ be the set of points $\tilde{x} \in \mathbb{R}^{n}$ such that $\alpha_{V}$ is not constant in any neighborhood of $\tilde{x}$. By definition of $\alpha_{V}$ it is seen that for a fixed $\tilde{x} \in \mathcal{S}$, and a small enough neighborhood $\mathcal{U}_{\widetilde{x}}$ of $\widetilde{x}$ (where $\alpha_{V}$ is not constant), we can find a finite family of disjoint open sets $\mathcal{V}_{i}$ such that $\alpha_{V}$ is constant on each $\mathcal{V}_{i}, \alpha_{V}(\widetilde{x})=\bigcup_{i} \alpha_{V}\left(\mathcal{V}_{i}\right)$ and $\mathcal{U}_{\widetilde{x}} \backslash \mathcal{S}=$ $\bigcup_{i} \mathcal{V}_{i}$. Hence, it follows from (9) and (11) that

$$
\partial V(\widetilde{x})=\overline{\operatorname{co}}\left\{\nabla V_{\ell}(\widetilde{x}): \ell \in \alpha_{V}(\widetilde{x})\right\} .
$$

The statement (10) indeed follows from (11) and (12).

\section{Linear Case}

For the linear differential inclusion

$$
\dot{x}(t) \in \overline{\mathrm{co}}\left\{A_{i} x(t) \mid i \in\{1, \ldots, m\}\right\},
$$

we can restrict our search for a Lyapunov function with degree of homogeneity 2 , and thus we can consider the maxmin function obtained from quadratic forms.

Definition 5. Given $K$ symmetric and positive definite matrices $P_{1}, \ldots P_{K} \in \mathbb{R}^{n \times n}$, the max-min of quadratics is defined as

$$
V(x)=\max _{j \in\{1, \ldots, J\}}\left\{\min _{k \in S_{j}}\left\{x^{\top} P_{k} x\right\}\right\},
$$

where $S_{j} \subset\{1, \ldots, K\}, \forall j \in\{1, \ldots, J\}$.

As a corollary to Theorem 1, we work out constructive conditions under which the max-min of quadratics is a Lyapunov function for (13). To rewrite inequalities (8) of Theorem 1 as bilinear matrix inequalities (BMI), we now recall how the multiple $\mathrm{S}$-procedure works. Let $P_{0}, P_{1}, \ldots, P_{K}$ be symmetric matrices. If $\exists \tau_{1}, \ldots, \tau_{K} \geq 0$ such that $P_{0}$ $\sum_{j=1}^{K} \tau_{j} P_{j}>0$ then

for each $x$ satisfying $x^{\top} P_{1} x \geq 0 \wedge \cdots \wedge x^{\top} P_{K} x \geq 0$, it holds that $x^{\top} P_{0} x>0$.

For a recent survey of the S-procedure, see [15]. We denote by $\mathbb{S}_{K}$ the group of all possible permutations of $K$ elements. We note that when we have $K$ quadratics $P_{1}, \ldots, P_{K}$, we can partition the space $\mathbb{R}^{n}$ as union of symmetric cones, that is $\mathbb{R}^{n}=\bigcup_{\rho \in \mathbb{S}_{K}} C_{\rho}$ where, given $\rho=\left(j_{1}, \ldots, j_{K}\right) \in \mathbb{S}_{K}$, we define

$$
C_{\rho}:=\left\{x \in \mathbb{R}^{n} \mid x^{\top} P_{j_{1}} x \leq \cdots \leq x^{\top} P_{j_{K}} x\right\} .
$$

It is observed that the map $\alpha_{V}$ is constant in the interior of $C_{\rho}$, so we write $\alpha_{V}\left(C_{\rho}\right)$ instead of $\alpha_{V}(x)$ for $x \in \operatorname{int}\left(C_{\rho}\right)$.

Corollary 1. Consider system (13) and the function $V$ in (14) described by the max-min of $K$ quadratic forms. If, for each $i \in\{1, \ldots, m\}$, and for each $\rho=\left(j_{1}, \ldots, j_{K}\right) \in \mathbb{S}_{K}$, there exist $\tau_{j_{1}}, \ldots, \tau_{j_{K-1}} \geq 0$ such that

$$
A_{i}^{\top} P_{\ell}+P_{\ell} A_{i}+\sum_{k=1}^{K-1} \tau_{j_{k}}\left(P_{j_{k+1}}-P_{j_{k}}\right)<0,
$$

$\forall \ell \in \alpha_{V}\left(C_{\rho}\right)$, then system (13) is GAS.

Proof. By the S-procedure, inequality (16) implies that for every nonzero $x \in C_{\rho}$

$$
x^{\top}\left(A_{i}^{\top} P_{\ell}+P_{\ell} A_{i}\right) x<0, \quad \forall \ell \in \alpha_{V}\left(C_{\rho}\right) .
$$


Since (16) holds for every $\rho \in \mathbb{S}_{K}$, by denoting $V_{j}(x)=$ $x^{\top} P_{j} x, j \in\{1, \ldots, K\}$, we get, for all $i \in\{1, \ldots, m\}$ and for all $x \in \mathbb{R}^{n}$,

$$
\nabla V_{\ell}(x)^{\top} A_{i} x<0, \quad \forall \ell \in \alpha_{V}(x) .
$$

The conditions of Theorem 1 are thus satisfied.

It is noted that, in general, since $\left|\mathbb{S}_{K}\right|=K$ !, finding a Lyapunov function for system (13) using (16) requires solving $m \cdot K$ ! inequalities, which involve $m(K-1) K$ ! nonnegative scalars and $K$ symmetric positive-definite matrices. It is clear that the computational burden grows quickly as function of $K$. We show in the next section that the required inequalities can be reduced for certain max-min functions.

\section{THREE QUADRATICS CASE}

In this section, we analyze some max-min functions of 3 quadratics defined by positive-definite and symmetric matrices $P_{1}, P_{2}$ and $P_{3}$. It can be taken as a simple useful model to underline some remarks and how the number of inequalities resulting from the S-procedure depends on the choice of the max-min composition. With an abuse of notation, we will write $\min \left\{P_{i}, P_{j}\right\}$ instead of $\min \left\{x^{\top} P_{i} x, x^{\top} P_{j} x\right\}$. The set $\operatorname{Mm}\left\{P_{1}, P_{2}, P_{3}\right\}$ has the following elements:

- Common Lyapunov function: $V=\max \left\{\min \left\{P_{i}\right\}\right\}$;

- Min of 2 quadratics: $V=\max \left\{\min \left\{P_{i}, P_{j}\right\}\right\}$;

- Max of 2 quadratics: $V=\max \left\{\min \left\{P_{i}\right\}, \min \left\{P_{j}\right\}\right\}$;

- Min of 3 quadratics: $V=\max \left\{\min \left\{P_{1}, P_{2}, P_{3}\right\}\right\}$;

- Max of 3 quadratics:

$$
V=\max \left\{\min \left\{P_{1}\right\}, \min \left\{P_{2}\right\}, \min \left\{P_{3}\right\}\right\}
$$

- Quasi-max functions:

$$
V=\max \left\{\min \left\{P_{1}\right\}, \min \left\{P_{2}, P_{3}\right\}\right\} ;
$$

- Quasi-min functions:

$$
V=\max \left\{\min \left\{P_{1}, P_{3}\right\}, \min \left\{P_{2}, P_{3}\right\}\right\} ;
$$

- Mid-of-quadratics function:

$$
V=\max \left\{\min \left\{P_{1}, P_{2}\right\}, \min \left\{P_{2}, P_{3}\right\} \min \left\{P_{1}, P_{3}\right\}\right\} .
$$

Our interest particularly lies in the last three cases because the remaining cases can be obtained more simply by considering maximum or minimum of $(3$ or less) quadratic functions. Moreover the cases of quasi-max and quasi-min are in some sense dual as we observe that $\max \left\{\min \left\{P_{i}, P_{k}\right\}, \min \left\{P_{j}, P_{k}\right\}\right\}=$ $\min \left\{P_{k}, \max \left\{P_{i}, P_{j}\right\}\right\}$.

\section{A. Comparison of Max Construction with Other Results}

Let us consider the max function $V=\max \left\{P_{1}, P_{2}, P_{3}\right\}$. Without loss of generality, we write down only the inequalities corresponding to the regions where $x^{\top} P_{3} x$ has the maximum value. We want to show that the two inequalities, corresponding to a fixed $i \in\{1, \ldots, m\}$, can be reduced to a single inequality, and hence the total computational burden can be reduced from $6 m$ to $3 m$ inequalities.

Lemma 2. Denote $A:=A_{i}$ for a fixed $i \in\{1, \ldots, m\}$. Consider the following statements:

$$
\text { (I } \begin{aligned}
& \exists \tau_{21}, \tau_{32} \geq 0 \text { such that } A^{\top} P_{3}+P_{3} A+\tau_{21}\left(P_{2}-P_{1}\right)+ \\
& \tau_{32}\left(P_{3}-P_{2}\right)<0 .
\end{aligned}
$$

(I $\left.I_{2}\right) \exists \tau_{12}, \tau_{31} \geq 0$ such that $A^{\top} P_{3}+P_{3} A+\tau_{12}\left(P_{1}-P_{2}\right)+$ $\tau_{31}\left(P_{3}-P_{1}\right)<0$.

(I $\left.I_{3}\right) \exists \lambda_{1}, \lambda_{2} \geq 0$ such that $A^{\top} P_{3}+P_{3} A+\lambda_{1}\left(P_{3}-P_{1}\right)+$ $\lambda_{2}\left(P_{3}-P_{2}\right)<0$.

Then, it holds that $\left(I_{1}\right) \wedge\left(I_{2}\right) \Longleftrightarrow\left(I_{3}\right)$.

Proof. $\left(I_{1}\right) \wedge\left(I_{2}\right) \Rightarrow\left(I_{3}\right)$. If $\tau_{21}=0$ then $\left(I_{3}\right)$ holds with $\lambda_{1}=0$ and $\lambda_{2}=\tau_{32}$. The case $\tau_{12}=0$ is analogous. If $\tau_{21} \neq 0, \tau_{12} \neq 0$ it suffices to multiply the inequality in item $\left(I_{1}\right)$ by $\frac{1}{\tau_{21}}$, then add it to the inequality given in $\left(I_{2}\right)$ multiplied by $\frac{1}{\tau_{12}}$ to arrive at $\left(I_{3}\right)$.

$\left(I_{3}\right) \Rightarrow\left(I_{1}\right) \wedge\left(I_{2}\right)$ : Let us take $\lambda_{1}$ and $\lambda_{2}$ such that $A^{\top} P_{3}+$ $P_{3} A+\lambda_{1}\left(P_{3}-P_{1}\right)+\lambda_{2}\left(P_{3}-P_{2}\right)<0$. We have

$$
\begin{aligned}
& A^{\top} P_{3}+P_{3} A+\lambda_{1}\left(P_{3}-P_{1}\right)+\lambda_{2}\left(P_{3}-P_{2}\right) \pm \lambda_{2} P_{1}= \\
& A^{\top} P_{3}+P_{3} A+\left(\lambda_{1}+\lambda_{2}\right)\left(P_{3}-P_{1}\right)+\lambda_{2}\left(P_{1}-P_{2}\right)<0,
\end{aligned}
$$

that is precisely the inequality in $\left(I_{2}\right)$. The inequality in $\left(I_{1}\right)$ can be derived with the same argument.

With this Lemma we have recovered the sufficient conditions for computing Lyapunov function via the max of quadratics, given in [3, Corollary 4.4], while using the more general framework of max-min functions.

\section{B. Mid of 3 Quadratics}

Let us consider the mid of quadratics described by

$$
V=\max \left\{\min \left\{P_{1}, P_{2}\right\}, \min \left\{P_{2}, P_{3}\right\}, \min \left\{P_{3}, P_{1}\right\}\right\} .
$$

We have called this function mid of quadratics because, for every $x \in \mathbb{R}^{n}$, it takes the value $x^{\top} P_{\ell} x$ such that $x^{\top} P_{j} x \leq$ $x^{\top} P_{\ell} x \leq x^{\top} P_{k} x$, where $i, k, \ell$ are different. Condition (16) in Corollary 1, for a fixed $i$, in this case becomes: $\exists \tau_{12}, \tau_{13}, \tau_{21}, \tau_{23}, \tau_{31}, \tau_{32}, \tilde{\tau}_{12}, \tilde{\tau}_{13}, \tilde{\tau}_{21}, \tilde{\tau}_{23}, \tilde{\tau}_{31}, \tilde{\tau}_{32} \geq 0$ such that

(123) $A_{i}^{\top} P_{2}+P_{2} A_{i}+\tau_{21}\left(P_{2}-P_{1}\right)+\tau_{32}\left(P_{3}-P_{2}\right)<0$,

(132) $A_{i}^{\top} P_{3}+P_{3} A_{i}+\tau_{31}\left(P_{3}-P_{1}\right)+\tau_{23}\left(P_{2}-P_{3}\right)<0$,

(213) $A_{i}^{\top} P_{1}+P_{1} A_{i}+\tau_{12}\left(P_{1}-P_{2}\right)+\tilde{\tau}_{31}\left(P_{3}-P_{1}\right)<0$,

(231) $A_{i}^{\top} P_{3}+P_{3} A_{i}+\tilde{\tau}_{32}\left(P_{3}-P_{2}\right)+\tau_{13}\left(P_{1}-P_{3}\right)<0$,

(312) $A_{i}^{\top} P_{1}+P_{1} A_{i}+\tilde{\tau}_{31}\left(P_{1}-P_{3}\right)+\tilde{\tau}_{21}\left(P_{2}-P_{1}\right)<0$,

(321) $A_{i}^{\top} P_{2}+P_{2} A_{i}+\tilde{\tau}_{23}\left(P_{2}-P_{3}\right)+\tilde{\tau}_{12}\left(P_{1}-P_{2}\right)<0$.

We have enumerated the inequalities using the triplets $\left(j_{1} j_{2} j_{3}\right)$, which correspond to the cone where $x^{\top} P_{j_{1}} x \leq$ $x^{\top} P_{j_{2}} x \leq x^{\top} P_{j_{3}} x$. This is the worst case: we can not regroup any inequalities, and $6 \mathrm{~m}$ inequalities involving $12 \mathrm{~m}$ non-negative scalars must be solved.

\section{Quasi-Max Function}

In this case, we consider the function described as

$$
V=\max \left\{\min \left\{P_{1}\right\}, \min \left\{P_{2}, P_{3}\right\}\right\} .
$$

The conditions given by (16), for a given $i \in$ $\{1, \ldots m\}$, are in this case: $\exists \tau_{12}, \tau_{13}, \tau_{21}, \tau_{23}, \tau_{31}, \tau_{32}$, $\tilde{\tau}_{12}, \tilde{\tau}_{13}, \tilde{\tau}_{21}, \tilde{\tau}_{23}, \tilde{\tau}_{31}, \tilde{\tau}_{32} \geq 0$ such that

(123) $A^{\top} P_{2}+P_{2} A+\tau_{21}\left(P_{2}-P_{1}\right)+\tau_{32}\left(P_{3}-P_{2}\right)<0$,

(132) $A^{\top} P_{3}+P_{3} A+\tau_{31}\left(P_{3}-P_{1}\right)+\tau_{23}\left(P_{2}-P_{3}\right)<0$,

(213) $A^{\top} P_{1}+P_{1} A+\tau_{12}\left(P_{1}-P_{2}\right)+\tilde{\tau}_{31}\left(P_{3}-P_{1}\right)<0$,

(231) $A^{\top} P_{1}+P_{1} A+\tilde{\tau}_{32}\left(P_{3}-P_{2}\right)+\tau_{13}\left(P_{1}-P_{3}\right)<0$,

(312) $A^{\top} P_{1}+P_{1} A+\tilde{\tau}_{31}\left(P_{1}-P_{3}\right)+\tilde{\tau}_{21}\left(P_{2}-P_{1}\right)<0$, 
(321) $A^{\top} P_{1}+P_{1} A+\tilde{\tau}_{23}\left(P_{2}-P_{3}\right)+\tilde{\tau}_{12}\left(P_{1}-P_{2}\right)<0$.

Reasoning as in Lemma 2 it easy to note that inequalities (231), (321), (213) are equivalent to the single inequality

$$
\exists \tilde{\lambda} \geq 0 \text { s.t. } A^{\top} P_{1}+P_{1} A+\tilde{\lambda}\left(P_{1}-P_{2}\right)<0 .
$$

This way, we can rewrite the sufficient conditions for the quasi-max Lyapunov function as: $\exists \tau_{21}, \tau_{23}, \tau_{31}, \tau_{32}, \tilde{\tau}_{21}, \tilde{\tau}_{31}, \tilde{\lambda} \geq 0$ such that

(123) $A^{\top} P_{2}+P_{2} A+\tau_{21}\left(P_{2}-P_{1}\right)+\tau_{32}\left(P_{3}-P_{2}\right)<0$,

(132) $A^{\top} P_{3}+P_{3} A+\tau_{31}\left(P_{3}-P_{1}\right)+\tau_{23}\left(P_{2}-P_{3}\right)<0$,

(312) $A^{\top} P_{1}+P_{1} A+\tilde{\tau}_{31}\left(P_{1}-P_{3}\right)+\tilde{\tau}_{21}\left(P_{2}-P_{1}\right)<0$,

(4) $A^{\top} P_{1}+P_{1} A+\tilde{\lambda}\left(P_{1}-P_{2}\right)<0$.

Note that, for every $i \in\{1, \ldots, m\}$, we have just one more inequality (involving just one more non-negative scalar) as compared to the max of quadratics case.

\section{An Illustrative Example}

Concluding this section, we consider an example introduced in [1] to show that existence of a common quadratic Lyapunov function is not necessary for asymptotic stability of a LDI. This example is also studied in [4, Example 2], where a max-of-quadratics Lyapunov functions is proposed.

Example 2. Let us consider the LDI problem

$$
\begin{aligned}
& \dot{x}(t) \in \overline{\operatorname{co}}\left\{A_{1} x(t), A_{2}(a) x(t)\right\}, \quad \text { where } \\
& A_{1}=\left[\begin{array}{cc}
-1 & -1 \\
1 & -1
\end{array}\right], \quad A_{2}(a)=\left[\begin{array}{cc}
-1 & -a \\
1 / a & -1
\end{array}\right],
\end{aligned}
$$

and $a>0$ is a scalar. It is proved in [1], using trajectorybased analysis, that the system admits a common quadratic Lyapunov function for $1<a<3+\sqrt{8}$. Here, we show how considering max-min candidate Lyapunov functions improves the estimates of the parameter $a$ for which the system is asymptotically stable. For simplicity in the table we have marked the maximal $a$ for which the set of BMI's corresponding to a particular max-min composition is feasible, that is the maximal $a$ for which we can prove stability using a particular type of functions.

\begin{tabular}{|l|l|l|l|}
\hline & CLF & Max of 2 & Min of 2 \\
\hline$a_{\max }$ & $3+\sqrt{8}$ & 8.10 & 6.78 \\
\hline \hline & Quasi-max & Quasi-min & Max of 3 \\
\hline$a_{\max }$ & 8.32 & 8.02 & 8.89 \\
\hline
\end{tabular}

Feasibility of BMIs has been checked with the help of the PENBMI solver for MATLAB. It turns out that, for this system, the choice of purely max function gives the best estimates of the parameter. In [4], it is shown that taking the max of 7 quadratics, one can prove stability until $a=10.108$.

\section{SWITCHING SYSTEMS}

We now focus our attention to system (2). Let $f_{1}, \ldots, f_{m} \in \mathcal{C}^{1}\left(\mathbb{R}^{n}, \mathbb{R}^{n}\right)$, the class of switching signals that we consider for system (2) is introduced in the following

Assumption 1. There exist finitely many connected sets $D_{1}, \ldots D_{N} \subset \mathbb{R}^{n}$ described as

$$
D_{j}:=\left\{x \in \mathbb{R}^{n} \mid S_{j}(x)>0 ; S_{j}: \mathbb{R}^{n} \rightarrow \mathbb{R} \text { is analytic }\right\},
$$

for $j=1, \ldots, N$, such that $\sigma$ is constant on each $D_{j}$, and $\bigcup_{j} \overline{D_{j}}=\mathbb{R}^{n}$, and $\bigcap_{j} D_{j}=\emptyset$.

Thus, given $f_{1}, \ldots, f_{m} \in \mathcal{C}^{1}\left(\mathbb{R}^{n}, \mathbb{R}^{n}\right)$ and $\sigma: \mathbb{R}^{n} \rightarrow$ $\{1, \ldots, m\}$ with Assumption 1, we can define a piecewise locally Lipschitz continuous function $f^{\mathrm{sw}}: \mathbb{R}^{n} \rightarrow \mathbb{R}^{n}$

$$
f^{\mathrm{sw}}(x)=f_{\sigma(x)}(x) .
$$

Definition 6. Given $f^{\mathrm{sw}}: \mathbb{R}^{n} \rightarrow \mathbb{R}^{n}$, and the system

$$
\dot{x}(t)=f^{\mathrm{sw}}(x(t)), \quad x(0)=x_{0}
$$

we define the set valued Filippov regularization

$$
\dot{x} \in F_{f^{\mathrm{sw}}}(x):=\bigcap_{\varepsilon>0} \bigcap_{\mu(\mathcal{N})=0} \overline{\mathrm{co}}\left\{f^{\mathrm{sw}}\left(B_{\varepsilon}(x) \backslash \mathcal{N}\right)\right\}
$$

where $\mu(\mathcal{N})$ is the Lebesgue measure of $\mathcal{N} \subset \mathbb{R}^{n}$. We say that $x: \mathbb{R} \rightarrow \mathbb{R}^{n}$ is a Filippov solution of system (18) if

1) $x$ is absolutely continuous, with $x(0)=x_{0}$,

2) $\dot{x}(t) \in F_{f^{\text {sw }}}(x(t))$ for almost all $t>0$.

For the vector field in (17), the computation of $F_{f^{\text {sw }}}$ simplifies as observed in [16], and is summarized below:

Proposition 1. Consider the vector field $f^{\mathrm{sw}}$ in (17) with $\sigma$ satisfying Assumption 1. Introduce the set-valued map $J$ : $\mathbb{R}^{n} \rightrightarrows\{1, \ldots, m\}$ as

$$
J(\widetilde{x}):=\left\{j \mid \forall \varepsilon>0, \exists x \in \mathbb{B}_{\varepsilon}(\widetilde{x}) \text { s.t. } \sigma(x)=j\right\} .
$$

It then holds that

$$
F_{f^{\text {sw }}}(x)=\overline{\operatorname{co}}\left\{f_{j}(x) \mid j \in J(x)\right\} .
$$

\section{A. General stability result}

Proposition 2. Consider system (2), and a switching law $\sigma: \mathbb{R}^{n} \rightarrow\{1, \ldots, m\}$ satisfying Assumption 1. Let us consider $K$ positive-definite and $\mathcal{C}^{1}$ functions $V_{1}, \ldots, V_{K}$ such that $V_{j}(0)=0 \forall j$. If, for a max-min function $V \in$ $\operatorname{Mm}\left\{V_{1}, \ldots, V_{K}\right\}$, and every $x \in \mathbb{R}^{n}$, there exists $\gamma \in \mathcal{K}$ such that

$$
\nabla V_{\ell}(x)^{\top} f \leq-\gamma(|x|), \quad \forall \ell \in \alpha_{V}(x), \forall f \in F_{f^{\text {sw }}}(x),
$$

then $V$ is a Lyapunov function for system (19).

Proof. The condition (21), as in the proof of Theorem 1 leads to

$$
v^{\top} f \leq-\gamma(|x|), \forall f \in F_{f^{\text {sw }}}(x), x \in \mathbb{R}^{n}
$$

for all $v \in \partial V(x)$, where $\partial V(x)$ is given in (12). Thus $V$ is a Lyapunov function for the Filippov regularization (19).

We underline that these conditions ensure the convergence to the origin even in the presence of the so-called sliding motion. If we can a priori rule out the sliding motion then requiring condition (21) is conservative, in the next subsection we propose stability conditions under this assumption.

\section{$B$. Linear switching systems with conic regions}

Given $A_{1}, \ldots, A_{m} \in \mathbb{R}^{n \times n}$, let us consider the linear switching system 


$$
\dot{x}=A_{\sigma(x)} x, \text { where } \sigma: \mathbb{R}^{n} \rightarrow\{1, \ldots, m\},
$$

where $\sigma$ satisfies Assumption 1 with $D_{j}$ given by

$$
D_{j}=\left\{x \in \mathbb{R}^{n} \mid x^{\top} Q_{j} x>0\right\},
$$

where $Q_{j}$ is a symmetric matrix, for $j \in\{1, \ldots, N\}$. We suppose that no sliding motion occurs along the switching surface, that is the set where $\sigma$ is not constant.

In order to provide a max-min Lyapunov function, homogenous of degree 2, we will choose positive-definite and symmetric matrices $P_{1}, \ldots, P_{K}$ such that resulting max-min function is non-differentiable only on the switching surfaces. In other words, we choose $P_{1}, \ldots, P_{K}$ such that, $\forall \rho \in \mathbb{S}_{K}$

$$
C_{\rho} \subset \bar{D}_{j}, \quad \text { for some } j \in\{1, \ldots, N\},
$$

where $C_{\rho}$ is defined by (15), and thus $\sigma$ takes a constant value in the interior of $C_{\rho}$ for every $\rho$, denoted by $\sigma\left(C_{\rho}\right)$.

Proposition 3. Consider system (22) satisfying Assumption 1 and $D_{j}$ satisfying (23). Let $P_{1}, \ldots, P_{K}>0$ be such that (24) holds for each $\rho \in \mathbb{S}_{K}$, and let $V$ be a max-min of quadratics $V$ as in (14). If, for each $\rho=\left(j_{1}, \ldots, j_{K}\right) \in \mathbb{S}_{K}$, there exist $\tau_{j_{1}}, \ldots, \tau_{j_{(K-1)}} \geq 0$ such that

$$
A_{\sigma\left(C_{\rho}\right)}^{\top} P_{\ell}+P_{\ell} A_{\sigma\left(C_{\rho}\right)}+\sum_{k=1}^{K-1} \tau_{j_{k}}\left(P_{j_{k+1}}-P_{j_{k}}\right)<0,
$$

for all $\ell \in \alpha_{V}\left(C_{\rho}\right)$, then $V$ is a Lyapunov function of (22).

Proof. Since there are no sliding motions, for every state trajectory $x(t)$ of the system (22) there exists a well defined sequence of switching time $0=t_{0}<t_{1}<t_{2}<\ldots,<t_{k} \ldots$ for which $\sigma(x(\cdot))$ is constant on the intervals $\left(t_{k-1}, t_{k}\right)$, for every $k \in \mathbb{N}$. Using S-procedure, we have, for each $x \in C_{\rho}$,

$x^{\top}\left(A_{\sigma\left(C_{\rho}\right)}^{\top} P_{\ell}+P_{\ell} A_{\sigma\left(C_{\rho}\right)}\right) x<-\gamma|x|^{2}$, for all $\ell \in \alpha_{V}(x)$

for some $\gamma>0$. Consider an interval $\left(t_{k-1}, t_{k}\right)$ we have

$$
V\left(x\left(t_{k}\right)\right)<\exp \left(-\delta\left(t_{k-1}-t_{k}\right)\right) V\left(x\left(t_{k-1}\right)\right) .
$$

Because $V$ decays exponentially between two consecutive switches, the result follows from [17, Theorem 3.1].

Example 1 Continued. We have already proved that there does not exist a convex Lyapunov function for the system (3). As every system trajectory "rotates" in the clockwise direction, so no motion occurs along the switching lines $\mathcal{S}_{12}, \mathcal{S}_{23}, \mathcal{S}_{31}$, see Fig. 1. Consider the matrices

$$
P_{1}=\left[\begin{array}{ll}
5 & 0 \\
0 & 1
\end{array}\right], \quad P_{2}=\left[\begin{array}{ll}
1 & 0 \\
0 & 5
\end{array}\right], \quad P_{3}=\left[\begin{array}{ll}
3 & 2 \\
2 & 3
\end{array}\right],
$$

and the max-min function

$$
V(x)=\max \left\{\min \left\{x^{\top} P_{1} x, x^{\top} P_{2} x\right\}, x^{\top} P_{3} x\right\} .
$$

We want to show that $V$ satisfies the conditions given in Proposition 3. We have to checking the inequalities:

1) $A_{2}^{\top} P_{2}+P_{2} A_{2}+\lambda_{1}\left(P_{2}-P_{3}\right)+\lambda_{2}\left(P_{1}-P_{2}\right)<0$,

2) $A_{1}^{\top} P_{1}+P_{1} A_{1}+\lambda_{3}\left(P_{1}-P_{3}\right)+\lambda_{4}\left(P_{2}-P_{1}\right)<0$,

3) $A_{3}^{\top} P_{3}+P_{3} A_{3}+\lambda_{5}\left(P_{3}-P_{1}\right)<0$,

4) $A_{3}^{\top} P_{3}+P_{3} A_{3}+\lambda_{6}\left(P_{3}-P_{2}\right)+\lambda_{7}\left(P_{1}-P_{3}\right)<0$.
Using numerical solvers (PENBMI for MATLAB), it follows that inequalities hold with $\bar{\lambda}=$ $(0.258,0.102,0.258,0.102,0.284,0.193,0.090), \quad$ so $\quad$ this max-min of quadratics is a Lyapunov function for the system (3) and hence, the system is GAS. A level set of this function is plotted in Fig. 1.

\section{CONCLUSiONS}

Considering the DI problem, we introduced a family of nonsmooth functions obtained by max-min combination. We proposed sufficient conditions under which an element of this family is a Lyapunov function. We also studied the utility of max-min functions for state-dependent switching systems. We illustrated stability using a max-min function by checking the feasibility of a set of BMIs. Further generalizations of stability conditions using max-min functions have been reported in [11]. Possible avenue for future research is the generalization of this approach to a wider class of systems, for example hybrid systems.

\section{REFERENCES}

[1] W.P. Dayawansa and C.F. Martin. A converse Lyapunov theorem for a class of dynamical systems which undergo switching. IEEE Transactions on Automatic Control, 44(4):751-760, 1999.

[2] A.P. Molchanov and Y.S. Pyatnitskiy. Criteria of asymptotic stability of differential and difference inclusions encountered in control theory. Systems \& Control Letters, 13(1):59-64, 1989.

[3] R. Goebel, A.R. Teel, T. Hu, and Z. Lin. Conjugate convex Lyapunov functions for dual linear differential inclusions. IEEE Transactions on Automatic Control, 51(4):661-666, 2006.

[4] R. Goebel, T. Hu, and A.R. Teel. Dual matrix inequalities in stability and performance analysis of linear differential/difference inclusions. In Current trends in nonlinear systems and control, pages 103-122. Springer, 2006.

[5] F. Blanchini and S. Miani. Set-Theoretic Methods in Control. Birkhäuser, 2008.

[6] A.A. Ahmadi, R.M. Jungers, P.A. Parrilo, and M. Roozbehani. Joint spectral radius and path-complete graph Lyapunov functions. SIAM Journal on Control and Optimization, 52(1):687-717, 2014.

[7] D. Angeli, N. Athanasopoulos, R.M. Jungers, and M. Philippe. Pathcomplete graphs and common Lyapunov functions. In Proc. 20th ACM Conf. Hybrid Systems: Computation and Control, pages 81-90, 2017.

[8] F. Blanchini and C. Savorgnan. Stabilizability of switched linear systems does not imply the existence of convex Lyapunov functions. Automatica, 44(4):1166-1170, 2008.

[9] T. Hu, L. Ma, and Z. Lin. Stabilization of switched systems via composite quadratic functions. IEEE Transactions on Automatic Control, 53(11):2571-2585, 2008.

[10] L. Xie, S. Shishkin, and M. Fu. Piecewise Lyapunov functions for robust stability of linear time-varying systems. Systems \& Control Letters, 31(3):165-171, 1997.

[11] M. Della Rossa, A. Tanwani, and L. Zaccarian. Max-Min Lyapunov Functions for Switched Systems and the Related Differential Inclusions. Submitted for publication, 2018.

[12] F.H. Clarke, Y.S. Ledyaev, R.J. Stern, and P.R. Wolenski. Nonsmooth analysis and control theory, volume 178 of Graduate Texts in Mathematics. Springer-Verlag, New York, 1998.

[13] F.M. Ceragioli. Discontinuous ordinary differential equations and stabilization. $\mathrm{PhD}$ thesis, Univ. Firenze, Italy, 2000. Available online: http://porto.polito.it/2664870/.

[14] F.H. Clarke. Optimization and nonsmooth analysis. Classics in Applied Mathematics. SIAM, 1990.

[15] I. Pólik and T. Terlaky. A survey of the S-lemma. SIAM review, 49(3):371-418, 2007

[16] J. Cortes. Discontinuous dynamical systems. IEEE Control Systems Magazine, 28(3):36-73, 2008.

[17] D. Liberzon. Switching in systems and control. Birkhaüser, 2003. 